\title{
Spinal nerve injury causes upregulation of ErbB2 and ErbB3 receptors in rat dorsal root ganglia
}

This article was published in the following Dove Press journal:

Journal of Pain Research

I February 2013

Number of times this article has been viewed

\author{
Satoshi Mizobuchil,* \\ Hirotaka Kanzaki ${ }^{1-3, *}$ \\ Hiroki Omiya' \\ Yoshikazu Matsuoka' \\ Norihiko Obata' \\ Ryuji Kaku' \\ Hirochika Nakajima' \\ Mamoru Ouchida ${ }^{2}$ \\ Kiyoshi Morita' \\ 'Department of Anesthesiology \\ and Resuscitology, ${ }^{2}$ Department \\ of Molecular Genetics, Okayama \\ University Graduate School \\ of Medicine, Dentistry and \\ Pharmaceutical Sciences, \\ Okayama University, Okayama, \\ Japan; ${ }^{3}$ Cedars-Sinai Medical \\ Center, Los Angeles, USA \\ *These authors contributed equally \\ to this work
}

Correspondence: Hirotaka Kanzaki Department of Anesthesiology and Resuscitology, Okayama University Graduate School of Medicine, Dentistry and Pharmaceutical Sciences, 2-5-I Shikata-cho, Okayama 700-8558, Japan

Tel +8I 862357327

Fax +8I 862357329

Email hirotaka.kanzaki@csmc.edu

\begin{abstract}
It is generally known that peripheral nerve injury causes changes in expression of some growth factors in the dorsal root ganglion. Altered expression of ErbB receptors, a wellknown growth factor in somatic cells, reportedly follows peripheral nerve injury in the spinal dorsal horn; however, it remains unknown whether the expression of these receptors is altered in the dorsal root ganglion after nerve injury. Therefore, this study examined the gene expression profiles of ErbB receptors in bilateral lumbar (L)4/L5 dorsal root ganglia, using L5-selective spinal nerve ligation in model rats as a peripheral nerve injury model. The expression of ErbB2 and ErbB3 was observed in the dorsal root ganglia of the mature rat, despite ErbB1 and ErbB4 showing only subtle expression. We also demonstrated that peripheral nerve injury induced significant increases in ErbB2 and ErbB3 in the ipsilateral dorsal root ganglion as compared with uninjured nerve. Expression changes in ErbB receptors appear to play important roles in nerve injury and subsequent nerve regeneration.
\end{abstract}

Keywords: DRG, spinal nerve ligation, SNL

\section{Introduction}

Injury to peripheral nerves initiates a sequence of cellular changes known as Wallerian degeneration or retrograde degeneration, resulting in marked neuroregeneration, and this contributes to the development of neuropathic pain, ${ }^{1,2}$ although the functional mechanisms remain to be elucidated. Understanding the changes in expression patterns of growth factors in the peripheral nerve system (PNS) and central nerve system (CNS) may be useful in understanding the mechanisms within neuroregeneration and neuropathic pain induced by peripheral nerve injury.

Neuregulins (NRGs) are known as a family of growth factors that have been identified to play a major role in the development and maintenance of the PNS and CNS. ${ }^{3}$ NRGs bind and activate a subfamily of the class I tyrosine kinase receptors known as the epidermal growth factor receptor (EGFR)/erythroblastic leukemia viral oncogene homolog (ERBB) family. There are four members in this family, ErbB1, ErbB2, ErbB3 and ErbB4. ${ }^{4,5}$ Previous studies have shown the importance of NRG1-induced activation of the ErbB receptors in the proper development of the nervous system. For example, large decreases have been shown by analysis of ErbB3 knockout mice. ${ }^{3}$ Ligand binding is relevant for promoting receptor dimer formation and activation of ErbB receptors through phosphorylation of the tyrosine-containing cytoplasmic domain of the receptor protein. ${ }^{4}$ ErbB receptors are activated by forming homodimeric and/or heterodimeric receptor complexes. Heterodimers that are known 
to form and result from the tyrosine phosphorylation, upon ligand binding, are ErbB1/2, 1/3, 2/3, 2/4, and 3/4. The ErbB2 receptor does not directly bind to NRG1 or any other ligands. Therefore, this receptor requires heterodimerization with ErbB3 or ErbB4 for phosphorylation induced by NRG1 binding and signal transduction. ${ }^{6,7}$

Given that our recent study demonstrated the alternative expression of NRG1 after spinal nerve injury, ${ }^{8}$ it is reasonable to hypothesize that the ErbB receptor family may act on the sensory and motor neurons through EGFR signaling, activated by ligand binding. If this hypothesis is correct, then these neurons should express one or more of the ErbB receptors that mediate ligand (eg, EGF and NRG1) actions. To test this, we performed a global analysis of the expression of ErbB receptors in adult rat dorsal root ganglions (DRGs). Because NRG1 expression potentially targeting DRG sensory neurons and/or spinal cord motor neurons is induced after peripheral nerve injury, it is possible that neuronal expression of ErbB receptors mediating NRG1 responses may also be altered after axotomy. Consequently, our studies investigated the changes in expression of ErbB receptors in DRG after spinal nerve injury by ligation.

\section{Materials and methods}

\section{Animals}

This study was approved by the Animal Care and Use Committee of Okayama University Medical School. Animals were treated in accordance with the Ethical Guidelines for the Investigation of Experimental Pain in Conscious Animals issued by the International Association for the Study of Pain. ${ }^{9}$

Adult male Wistar rats (CLEA Japan Inc, Tokyo, Japan) weighing 190-220 g at the time of surgery were used. Animals were housed individually in cages, under a 12-hour light/dark cycle, with free access to food and water. The experimental rats were allowed to acclimatize to the facility for 5 to 7 days prior to surgery.

\section{Nerve injury model}

As the nerve injury model, a lumbar (L)5 spinal nerve ligation (L5SNL) model was used. All the rats $(n=7)$ were placed under anesthesia with pentobarbital sodium $(40 \mathrm{mg} / \mathrm{kg}$ intraperitoneally). Additional inhalation anesthesia, with $1.5 \%-2 \%$ of isoflurane in $100 \%$ oxygen, was given as needed. The L5SNL was performed using a modification of the procedure described previously. ${ }^{8}$ Briefly, the left transverse process of the L6 vertebra was gently removed, and the left L5 spinal nerve was exposed and tightly ligated with a 6-0 silk thread, with care being taken to avoid trauma to the nearby L4 spinal nerve. Sham surgery animals were prepared as controls for the SNL model, in an identical manner but without injuring the nerve.

Complete Freund's adjuvant (CFA) was used in the preparation of a peripheral inflammation model (CFA model, $n=6$ ), which was used as a non-nerve injury hyperalgesia control model. For this model, animals were treated with a subcutaneous injection of $100 \mu \mathrm{L}$ of CFA $(0.1 \%$ heat-killed Mycobacterium tuberculosis; Sigma-Aldrich, St Louis, MO, USA) into the plantar surface of the left hind paw, under ether anesthesia. "Sham" animals $(n=6)$ were injected with saline instead of CFA and used as controls for the CFA model. Naïve animals $(n=13)$ were used as negative controls for both the SNL and CFA models.

\section{Behavioral assessment}

The von-Frey test was performed to validate the model rats. For assessment of tactile allodynia, the sensitivity to mechanical stimulus was tested by determining the hind paw withdrawal response to von Frey filaments (TouchTest $^{\circledR}$ Sensory Evaluator, Stoelting Co, Wood Dale, IL, USA). Rats were placed individually in a plastic cage $\left(13 \times 10 \times 15 \mathrm{~cm}^{3}\right)$ with an elevated wire mesh bottom, allowing full access to the plantar surface of both hind paws. Mechanical stimuli were applied to the medial plantar aspect of each hind paw with one of a series of nine von-Frey filaments $(0.4,0.6,1.0,1.4,2.0,4.0,6.0,8.0$, and $15.0 \mathrm{~g})$. Each trial was started with a von Frey force of $2 \mathrm{~g}$ for 1-2 seconds. Stimuli were presented at intervals of at least 10 seconds, allowing for the apparent resolution of any behavioral responses to the previous stimuli. On the basis of the response pattern and the force of the final filament, the 50\% paw withdrawal threshold (PWT) was determined using the up-down method of Dixon ${ }^{10}$ and calculated using the formula described by Chaplan et al. ${ }^{11}$ If the strongest filament did not elicit a response, the threshold was recorded as $15.0 \mathrm{~g}$. For the SNL model and negative controls, the tests were done before surgery and 1-14 days after surgery. For the CFA model and negative controls, tests were conducted before the injection and on the third day after the injection.

After carrying out the behavioral assessment, rats were killed by decapitation under deep anesthesia, using an intraperitoneal pentobarbital overdose (60 mg/kg). Bilateral L4/L5 DRGs were dissected rapidly and frozen immediately. DRGs were stored at $-80^{\circ} \mathrm{C}$ until use. 


\section{Quantitative real-time polymerase chain reaction (PCR) analysis}

Total RNA samples were prepared using standard acidphenol extraction procedures with ISOGEN (Nippon Gene Co, Ltd, Tokyo, Japan).

Complementary (c)DNA was synthesized by reverse transcription from $2 \mu \mathrm{g}$ of each total RNA sample, with ReverTra Ace ${ }^{\circledR}$ First Strand cDNA Synthesis Kit (Toyobo Co, Ltd, Osaka, Japan). Templates were amplified in $20 \mu \mathrm{L}$ of reaction mixture containing $10 \mu \mathrm{L}$ of Power SYBR ${ }^{\circledR}$ Green PCR Master Mix (Life Technologies, Carlsbad, CA, USA), 7.2 $\mu \mathrm{L}$ of DNasefree water, $0.2 \mu \mathrm{M}$ each of forward and reverse primer, and $2 \mu \mathrm{L}$ of cDNA solution. The sequences of forward and reverse primers for the EGFR, ErbB2, ErbB3, ErbB4, and the glyceraldehyde 3-phosphate dehydrogenase $(G A P D H)$ gene are shown in Table 1. Quantitative real-time polymerase chain reaction (PCR) was performed with the 7300 Real Time PCR system (Life Technologies), with the following amplification conditions: $95^{\circ} \mathrm{C}$ for 10 minutes, followed by 40 cycles of 30 seconds at $95^{\circ} \mathrm{C}$ and 2 minutes at $59^{\circ} \mathrm{C}$. Quantification was done by the Comparative Ct method, using the 7300 Real Time PCR System with Sequence Detection Software version 1.4 (Life Technologies). The cDNA amount in each sample was normalized to the crossing point of the housekeeping gene $G A D P H$. These assays were repeated twice in duplicate. All of the PCR products were validated by melting curve analysis.

\section{Western blot analysis}

An L5 DRG from each of the groups was homogenized in $100 \mu \mathrm{L}$ of radio-immunoprecipitation assay (RIPA) lysis buffer $(50 \mathrm{mM}$ Tris- $\mathrm{HCl}$ [pH 8.0], $150 \mathrm{mM}$ sodium chloride, $1.0 \%$ Igepal CA-630, 0.5\% sodium deoxycholate, $0.1 \%$ sodium dodecyl sulfate [SDS]) (Sigma-Aldrich) with protease inhibitor cocktail Cat\# P8340 (Sigma-Aldrich) and phosphatase inhibitor cocktail 2 Cat\# P5726 (Sigma-Aldrich). Lysates were centrifuged at $15,000 \times \mathrm{g}$ for 15 minutes at $4^{\circ} \mathrm{C}$, and the protein concentration was determined by bicinchoninic acid (BCA) protein assay reagent (Thermo Fisher Scientific, Waltham, MA, USA). Western blot analysis was performed according to the guidelines of the Trans-Blot Turbo Transfer system protocol (Bio-Rad Laboratories Inc, Hercules, CA, USA). In brief, $40 \mu \mathrm{g}$ of total proteins was heated for 5 minutes at $95^{\circ} \mathrm{C}$ and then separated on 4\%-20\% SDS-polyacryl-amide gel and electrotransferred to a nitrocellulose membrane (BIO-RAD Laboratories Inc). Visualization of the protein band was performed with a standard enhanced chemiluminescence (ECL) detection system. Membranes were blocked in 5\% of nonfat dry milk in Tris-bufferd saline-Tween 20 (TBS-T) buffer, and then incubated over night at $4^{\circ} \mathrm{C}$ with the following primary antibodies: 1:100 rabbit polyclonal anti-ERBB2 (sc-284: Santa Cruz Biotechnology Inc, Santa Cruz, CA, USA), 1:200 rabbit polyclonal anti-ERBB3 (sc-285, Santa Cruz Biotechnology, Inc) or 1:100,000 mouse monoclonal anti-GAPDH (G8795, Sigma-Aldrich). After washing with TBS-T, membranes were incubated with peroxidase-conjugated secondary antibody in TBS-T for 1 hour at room temperature. Blots were washed and hybridization signals were measured by ECL detection using the ECL Plus Western Blotting Detection System (Life Technologies, Grand Island, NY, USA) and ChemiDoc XRS System (BIO-RAD Laboratories Inc). Images were analyzed quantitatively using ImageJ 1.46r image analysis software.

\section{Statistical analysis}

All values are expressed as mean or mean \pm standard deviation (SD). For the data of behavioral tests, tactile withdrawal thresholds with von Frey filaments are nonparametric variables. Between testing days, the data were analyzed using Friedman analysis of variance (ANOVA) for repeated measurements, followed by Wilcoxon matched pairs signed rank test, when appropriate. For the quantitative real-time PCR data, statistical significance was determined by the paired $t$-test. $P<0.05$ was considered statistically significant.

\section{Results}

\section{Behavioral assessment of L5SNL} rats for validation of hyperalgesia

L5SNL model rats are well-established as a neuropathic pain model that shows hyperalgesia, without phenomena such as

Table I Oligonucleotide sequences of primers for real-time RT-PCR

\begin{tabular}{lllll}
\hline Target cDNA & Accession no & Forward (5' to 3') & Reverse (5' to 3') & Amplicon (bp) \\
\hline ErbBI/Egfr & NM_03I507 & tccctt tggagaacctgc ag & tcgcacagcaccgatcagaa & 152 \\
ErbB2 & NM_017003 & gagcttctctccacatgacc & taaaggaggctgaggctgaa & 157 \\
ErbB3 & NM_0172I8 & cgtcatgccagatacacacc & agggctaccccttctcttcc & 148 \\
ErbB4 & NM_021687 & aggctacatgactcccatgc & gctggaagcgctgtgatact & 136 \\
GAPDH & NM_017008 & gacaactttggcatcgtgga & atgcagggatgatgttctgg & 133 \\
\hline
\end{tabular}

Abbreviations: RT-PCR, reverse transcription polymerase chain reaction; CDNA, complementary DNA; EGFR, epidermal growth factor receptor. 
paralysis, when spinal nerve ligation/injury is successfully induced. ${ }^{12}$ To investigate hyperalgesia in L5SNL rats, we performed behavioral assessments with the von-Frey test before and after L5SNL. We used a CFA model, which shows hyperalgesia as a non-nerve injury. ${ }^{13}$ Mechanical allodynia was observed in L5SNL model rats throughout the examination period (days 1-14 after L5SNL). The CFA injection induced allodynia at 3 days after treatment. Prior to SNL and intraplantar injection of CFA, there were no differences in $50 \%$ PWT on the bilateral hind paws (ipsilateral 13.6 $\pm 1.8 \mathrm{~g}$ vs contralateral $13.6 \pm 1.8 \mathrm{~g}$; ipsilateral $15.0 \pm 0 \mathrm{~g}$ vs contralateral $15.0 \pm 0 \mathrm{~g}$, respectively). The $50 \%$ PWTs in the SNL group were $3.1 \pm 0.8,2.8 \pm 0.9,2.5 \pm 1.4,3.2 \pm 1.2,2.1 \pm 0.4$, and $2.5 \pm 1.0 \mathrm{~g}$ at $1,3,7,9,11$, and 14 days after surgery, respectively (Figure 1A). Significantly decreased 50\% PWTs were observed from days 1 through 14 after surgery. In the CFA group, the ipsilateral 50\% PWT was also significantly lower on days 1 and 3 (2.8 $\pm 1.8 \mathrm{~g}$ and $4.2 \pm 3.8 \mathrm{~g}$, respectively) (Figure 1B). There were no changes in the $50 \%$ PWT on the contralateral side in any of all the groups. There were also no changes in $50 \%$ PWT on either the ipsilateral or contralateral sides in the sham and naïve groups. The model rats were thus validated based on these results.

\section{Spinal nerve injury increased the expression of ErbB2 and ErbB3 in the DRG}

The expression profiles of ErbB receptors were examined by quantitative real-time PCR in ipsilateral and contralateral
L4/L5 DRGs of the SNL model, sham-SNL, and naïve rats (Figure 2). Expression of ErbB2 and ErbB3 was observed in both L4/L5 DRGs of mature naïve rats, despite the subtle expression of ErbB1 and ErbB4. The expression of ErbB2 and ErbB3 was significantly elevated in ipsilateral L5 DRGs after spinal nerve injury (199\% of the contralateral value, $P=0.0019$; and $196 \%, P=0.0005$, respectively) (Figure 2 ). In contrast, the expression of ErbB receptors was not altered in ipsilateral L4 DRGs after spinal nerve injury. No expression changes were observed in the negative controls, sham-SNL, CFA, or sham-CFA (Figures 2 and 3). ErbB3 overexpression was also confirmed at the protein level, by Western blot (229\% of contralateral value, $P=0.0020)$, while ErbB2 expression could not be quantified due to the low protein abundance (Figure 4). This result is consistent with a finding that the expression of ErbB3 is higher than ErbB2 at mRNA (Figures 2 and 4).

\section{Time course of ErbB2 and ErbB3 expression after spinal nerve injury}

We also investigated the time-dependent expression changes in expression ErbB receptors. Significantly increased expression of ErbB2 and ErbB3 was observed in the ipsilateral L5 DRGs at day 14, whereas the expression levels did not change until day 7 (Figure 5). Sham surgery did not induce any changes in ErbB receptor expression in either L4/L5 DRGs at the mRNA level. Furthermore, basal expression of each gene was not altered. The time course of ErbB2 and ErbB3 upregulation was not consistent with behavioral
A

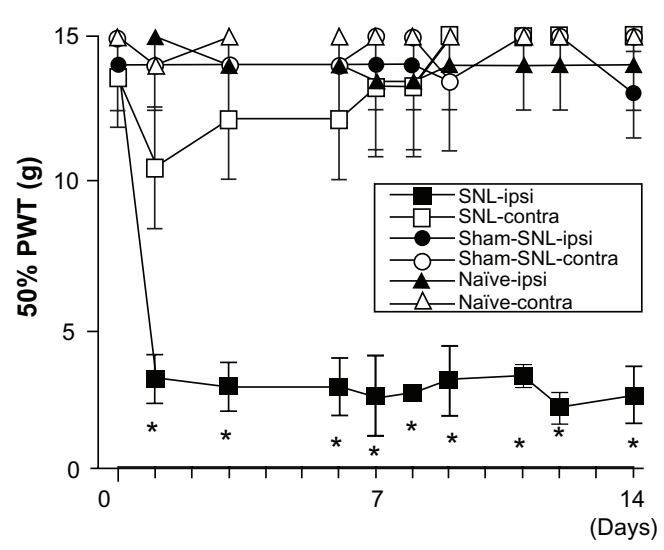

B

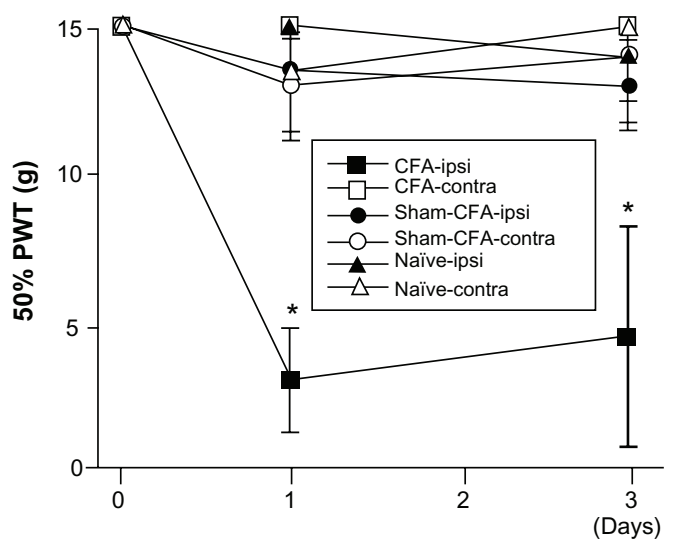

Figure I (A) Behavioral assessment of the L5 spinal nerve ligation model $(n=7)$. There was no significant difference in $50 \%$ PWT between the ipsilateral and contralateral PWT of the sham group or between the contralateral sides of the SNL and sham groups; (B) behavioral assessment of the CFA model rats ( $n=6$ ). There was no significant difference in 50\% PWT between the ipsilateral and contralateral PWT of the sham group or between the contralateral sides of the CFA and sham groups.

Notes: The mechanical sensitivity of the hind paw was determined as the $50 \%$ PWT. Data are mean \pm SD, $* P<0.05$ (A) vs the contralateral PWT of the SNL model; (B) vs the contralateral PWT of the CFA model rat.

Abbreviations: L, lumbar; SNL, spinal nerve ligation; PWT, paw withdrawal threshold; CFA, complete Freund's adjuvant; SD, standard deviation; Ipsi, ipsilateral side; Contra, contralateral side. 
A ErbB1/EGFR

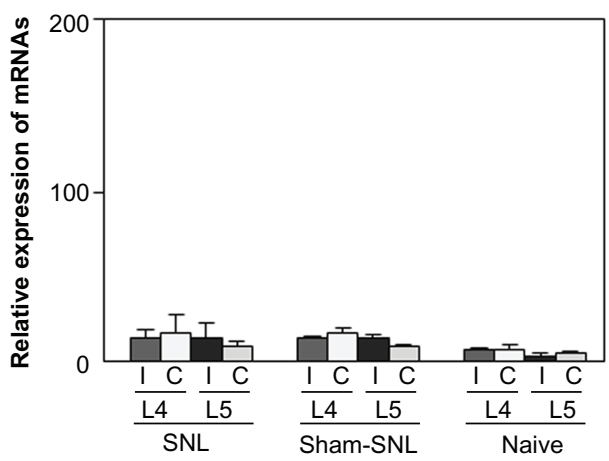

C ErbB3

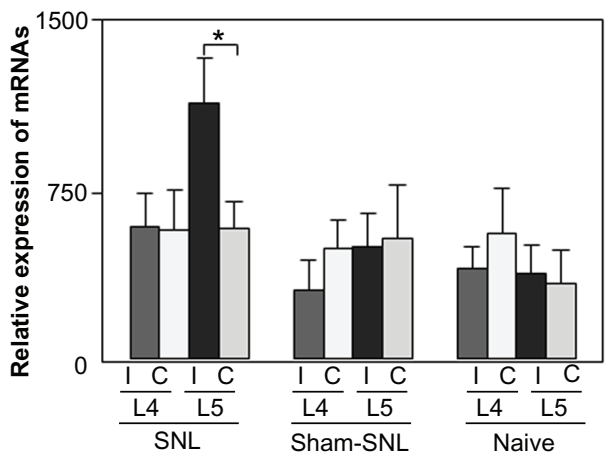

B ErbB2

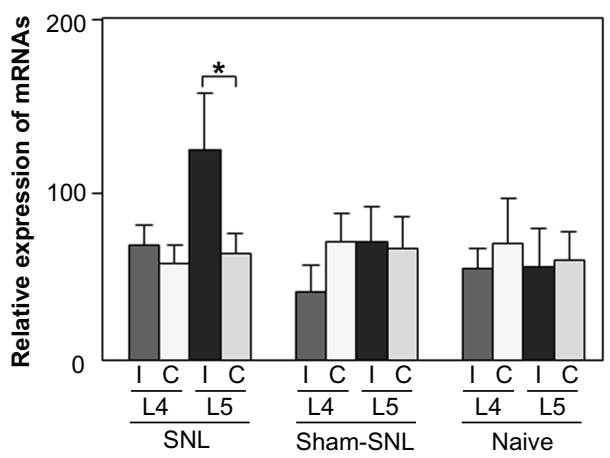

D ErbB4

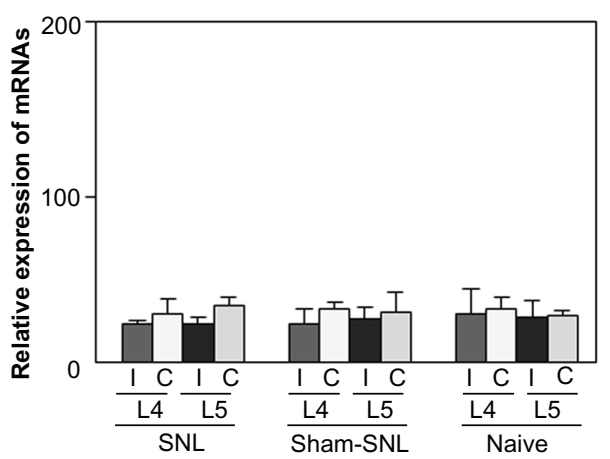

Figure 2 Expression of EGFR, ErbB2, ErbB3, and ErbB4 transcripts in the bilateral L4 and L5 DRG of SNL model rats, sham-SNL rats and naïve rats. Expression of the (A) EGFR; (B) ErbB2; (C) ErbB3; and (D) ErbB4 mRNA at day I4; all of the expression levels were normalized to that of GAPDH.

Notes: Data are mean \pm SD. SNL group and sham-SNL group; $n=7$, naïve group; $n=13, * p<0.05$ vs the contralateral DRG of the SNL group.

Abbreviations: EGFR, epidermal growth factor receptor; ErbB, erythroblastic leukemia viral oncogene homolog; L, lumbar; DRG, dorsal root ganglion; SNL, spinal nerve ligation; GAPDH, glyceraldehyde 3-phosphate dehydrogenase gene; SD, standard deviation; I, ipsilateral side; C, contralateral side.

development (Figure 1A). These data suggest that upregulation of ErbB2 and ErbB3 in the L5 DRG is correlated with the delayed event to response to spinal nerve injury.

\section{Discussion}

ErbB receptors are known to be involved in the proper development of the nervous system. ${ }^{14-17}$ Recently, ErbB2 has been reported to be upregulated in the dorsal horn by spinal nerve injury. ${ }^{18}$ However, the expression profiles of ErbB receptors in adult rat DRGs and their alteration after nerve-ligationcaused injury remain unknown, while the expression of ErbB2, ErbB3, and ErbB4 in intact rat DRG was confirmed. ${ }^{19}$ In addition to this fact, NRG1, a ligand of ErbB receptors, has been identified to play a major role in the development and maintenance of the PNS and CNS. ${ }^{3}$ Therefore, we hypothesized that adult DRG neurons express the ErbB receptors necessary for NRG1 responsiveness and that its altered expression may be induced by nerve injury. To test our hypothesis, we examined the basal expression profiles of the ErbB receptor in adult rat DRGs and investigated whether spinal nerve injury induced changes in the expression of ErbB receptors.
We observed that a subset of the ErbB receptors, ErbB2 and ErbB3, were expressed in both L4 and L5 DRGs under uninjured conditions and were significantly altered by peripheral nerve injury in the L5 DRG, while ErbB1 and ErbB4 were weakly expressed and not altered by nerve injury. Consistent with our hypothesis, the upregulated ErbB2 and ErbB3 receptors are known as a subset of ErbB receptors that require NRG1 for activation.

In order to determine whether hyperalgesia induces changes in ErbBs in the DRG, a CFA model was used as a hyperalgesia model without nerve injury and was compared with the SNL model, which shows hyperalgesia with nerve injury (Figure 1). The patterns of ErbB expression in the L4/L5 DRGs in the CFA model were the same as those in naïve rat; thus, hyperalgesia is not directly related to the upregulation of ErbB2 and ErbB3 in DRGs. In the rats without nerve injury, no differences in ErbB expression in the L4/L5 DRGs were observed, and ErbB2 and ErbB3 were intensely expressed, while the expression of ErbB1 and ErbB4 was modest. In contrast to the general understanding of the importance of ErbB4 functions in the $\mathrm{CNS},{ }^{20}$ our findings suggest that ErbB2 and ErbB3 play important roles 
A ErbB1/EGFR

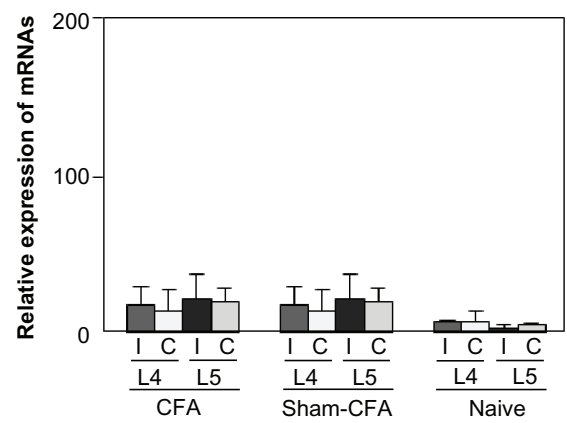

C ErbB3

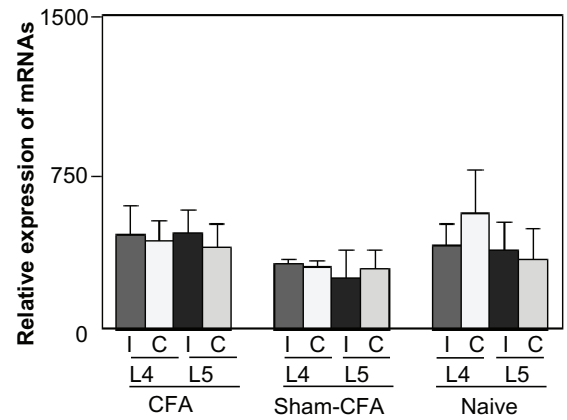

B ErbB2

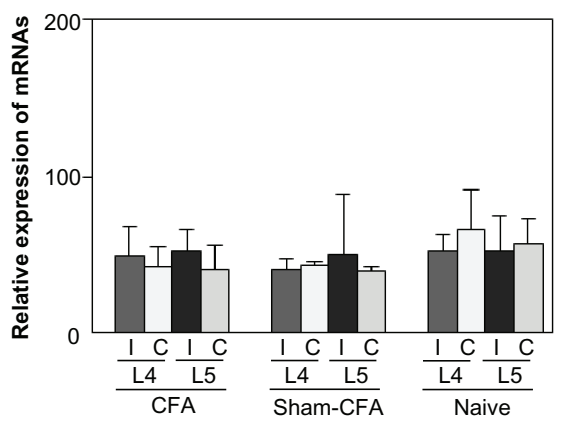

D ErbB4

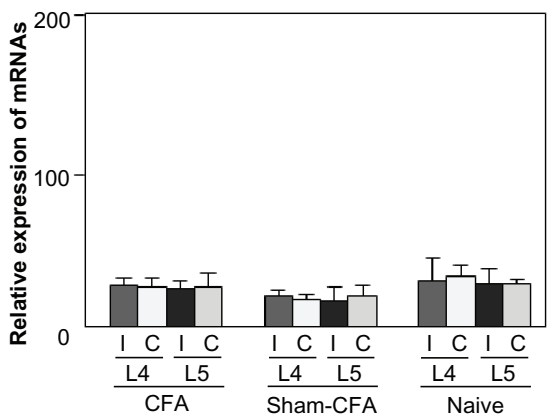

Figure 3 Ratio of expression of the EGFR, ErbB2, ErbB3, and ErbB4 transcripts in the bilateral L4 and L5 DRG of CFA model rats, sham-CFA rats and naive rats. Expression of the (A) EGFR; (B) ErbB2; (C) ErbB3; and (D) ErbB4 mRNA; all of the expression levels were normalized to that of GADPH.

Notes: Data are mean \pm SD. CFA group and sham-CFA group: $n=6$; naïve group: $n=13$.

Abbreviations: EGFR, epidermal growth factor receptor; ErbB, erythroblastic leukemia viral oncogene homolog; L, lumbar; DRG, dorsal root ganglion; CFA, complete Freund's adjuvant; GAPDH, glyceraldehyde 3-phosphate dehydrogenase gene; SD, standard deviation; I, ipsilateral side; C, contralateral side.

in the PNS through its compartment-specific expression, while the functional mechanisms of tissue- or cell-specific expression and localization remain to be elucidated in further studies. Nevertheless, as the actions of NRG1 are, instead, mediated when this factor binds to ErbB3 or ErbB4, with
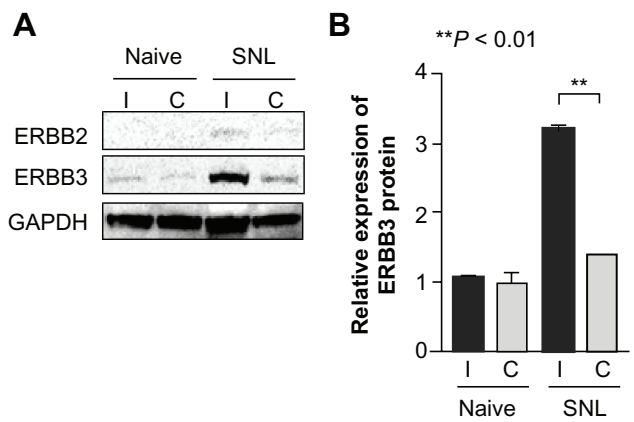

Figure 4 Expression of ERBB2 and ERBB3 proteins in the bilateral L5 DRG of SNL model and naïve rats. (A) ErbB2 and ErbB3 proteins were determined by Western blot at day 14. GAPDH was used as a loading control; (B) Relative expression of ERBB3 was calculated by the formula: Int $\mathrm{t}_{\text {target }} / \operatorname{lnt} \mathrm{t}_{\text {naive-contralateral }}=\operatorname{lnt}_{\mathrm{ERBB} 3} / \operatorname{lnt} \mathrm{GAPDH}_{\mathrm{GP}}$. Notes: Data are mean $\pm S D$. $* * P<0.01$ statistically significant when compared with the contralateral DRG of each groups using one-way ANOVA, followed by Scheffe's F-test.

Abbreviations: ErbB, erythroblastic leukemia viral oncogene homolog; L, lumbar; SNL, spinal nerve ligation; GAPDH, glyceraldehyde 3-phosphate dehydrogenase gene; Int, Intensity; SD, standard deviation; ANOVA, analysis of variance; C, contralateral side; I, ipsilateral side; SNL, spinal nerve ligation.
ErbB2 activation occurring when the kinase subsequently heterodimerizes with either of these occupied receptors, ${ }^{21}$ our findings suggest that the ErbB2/ErbB3 heterodimer is related to PNS homeostasis.

We also clearly demonstrated that nerve injury from SNL induced significant increases in ErbB2 and ErbB3 in the injured DRG (Figures 2 and 4). This phenomenon was observed from 14 days after nerve injury, whereas the time course of ErbB2 and ErbB3 upregulation was not consistent with behavioral development (Figures 1A and 5). Furthermore, from our data using the CFA model, transient inflammation occurring remotely from the DRG, with associated mechanical allodynia, is not correlated with aberrant expression of ErbB receptors. These findings of injuredDRG-specific increases in ErbB2 and ErbB3 suggest that upregulation of ErbB2 and ErbB3 in L5 DRG is correlated with the delayed response to spinal nerve injury (eg, nerve regeneration and late-onset chronic pain). In contrast with our results, Pearson and Carroll ${ }^{22}$ reported that DRG expression of ErbB2 and ErbB3 was modestly decreased after sciatic nerve transection, at 1 week after surgery, and that expression was regained in after a further week. These dissimilar findings raise the possibility of differences in the type of neural 


\section{A ErbB1/EGFR}

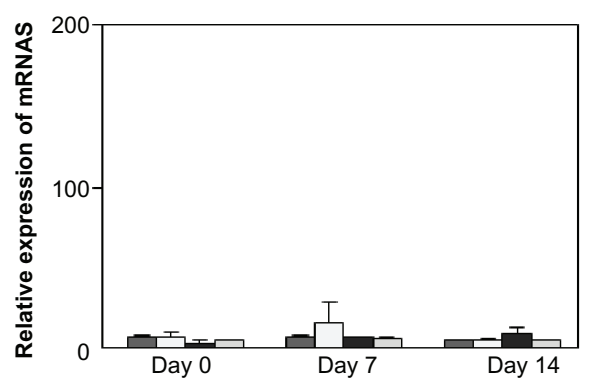

C ErbB3

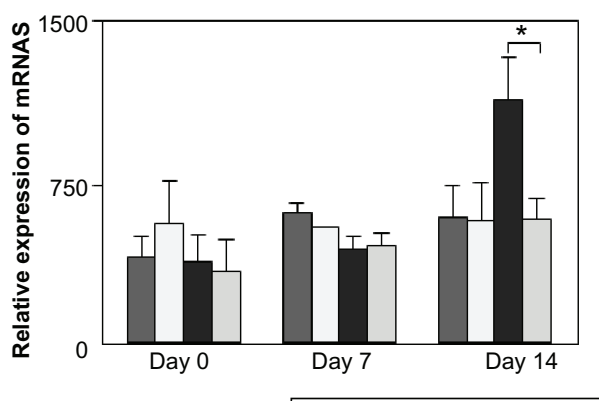

B ErbB2

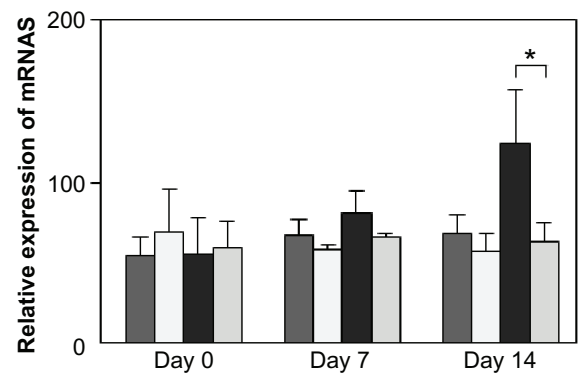

D ErbB4

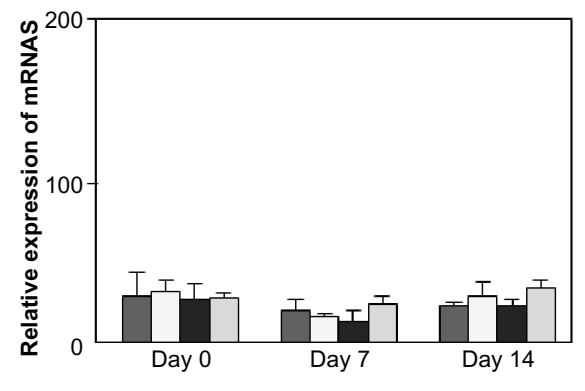

$\square$ L4-ipsi $\square$ L4-contra $\square$ L5-ipsi $\square$ L5-contra

Figure 5 Time course and the expression of ErbBs in bilateral L4/L5 DRG of SNL model rats. Expression of the (A) EGFR (B) ErbB2 (C) ErbB3 (D) ErbB4 mRNA; expressions were tested before surgery and at 7 and 14 days after surgery.

Notes: All of the expression levels were normalized to that of the GADPH. Data are mean \pm SD $(n=7)$. *P $<0.05$ vs contralateral side.

Abbreviations: ErbB, erythroblastic leukemia viral oncogene homolog; L, lumbar; DRG, dorsal root ganglion; SNL, spinal nerve ligation; GAPDH, glyceraldehyde 3-phosphate dehydrogenase gene; SD, standard deviation; EGFR, epidermal growth factor receptor; Ipsi, ipsilateral side; Contra, contralateral side.

damage arising from transection and ligation. Persistent neural damage caused by SNL may lead to continuous increases in ErbB2 and ErbB3 expression in a delayed manner.

Similar to the receptor expression in the DRG, significant increases in ErbB2 and ErbB3 in dorsal horn cells and neurons are induced by nerve injury, and corresponding enhancement of pain behavior has been also observed. ${ }^{18,22}$ Furthermore, Matsuoka and Yang ${ }^{23}$ have suggested that the MAPK/ERK signaling pathway, one of the main pathways of ErbB signaling, was activated in the DRG of nerve injury model rat. Taken together, our data suggest that activation of ErbB signaling, which may be induced by ErbB2/3 heterodimerization, is correlated with nerve injury and is a potential therapeutic target of nerve-injury-induced neurological lesions (eg, neuropathic pain). Our data indicate that the ErbB2/3 heterodimer functions in the ipsilateral DRG after nerve injury, but this requires confirmation by further studies at the protein level and observation of long-term outcomes.

\section{Conclusion}

In this study, we investigated the expression of ErbB2 and ErbB3 in rat DRGs. Spinal nerve injury induces overexpression of the ErbB2 and ErbB3 receptors in the L5
DRG. Nevertheless, the mechanisms by which these receptors are upregulated and the cells from which these receptors are derived (eg, sensory neuron, motor neuron, glia) remain to be elucidated in further studies. Our findings demonstrate that spinal nerve injury induces upregulation of the ErbB receptors in the DRG, and this may be of major importance in the study of neurogenesis in mature mammals.

\section{Acknowledgments}

This study was supported by JSPS KAKENHI Grant \#21592009. The authors thank E Suzuki and T Mizuno, Department of Biomedical Sciences, Cedars-Sinai Medical Center, for helpful discussion.

\section{Disclosure}

There are no conflicts of interest associated with this work.

\section{References}

1. Atanasoski S, Scherer SS, Sirkowski E, et al. ErbB2 signaling in Schwann cells is mostly dispensable for maintenance of myelinated peripheral nerves and proliferation of adult Schwann cells after injury. $J$ Neurosci. 2006;26(7):2124-2131.

2. Calvo M, Bennett DL. The mechanisms of microgliosis and pain following peripheral nerve injury. Exp Neurol. 2012;234(2):271-282. 
3. Adlkofer K, Lai C. Role of neuregulins in glial cell development. Glia. 2000;29(2):104-111.

4. Carraway KL 3rd, Cantley LC. A neu acquaintance for erbB3 and erbB4: a role for receptor heterodimerization in growth signaling. Cell. 1994;78(1):5-8.

5. Ullrich A, Schlessinger J. Signal transduction by receptors with tyrosine kinase activity. Cell. 1990;61(2):203-212.

6. Carraway KL 3rd, Sliwkowski MX, Akita R, et al. The erbB3 gene product is a receptor for heregulin. J Biol Chem. 1994;269(19):14303-14306.

7. Tzahar E, Levkowitz G, Karunagaran D, et al. ErbB-3 and ErbB-4 function as the respective low and high affinity receptors of all Neu differentiation factor/heregulin isoforms. J Biol Chem. 1994; 269(40):25226-25233.

8. Kanzaki H, Mizobuchi S, Obata N, et al. Expression changes of the neuregulin 1 isoforms in neuropathic pain model rats. Neurosci Lett. 2012;508(2):78-83.

9. Zimmermann M. Ethical guidelines for investigations of experimental pain in conscious animals. Pain. 1983;16(2):109-110.

10. Dixon WJ. Efficient analysis of experimental-observations. Anпu Rev Pharmacol Toxicol. 1980;20:441-462.

11. Chaplan SR, Bach FW, Pogrel JW, Chung JM, Yaksh TL. Quantitative assessment of tactile allodynia in the rat paw. J Neurosci Meth. 1994;53(1):55-63.

12. Kim SH, Chung JM. An experimental model for peripheral neuropathy produced by segmental spinal nerve ligation in the rat. Pain. 1992;50(3):355-363.

13. Taurog JD, Argentieri DC, McReynolds RA. Adjuvant arthritis. Methods Enzymol. 1988;162:339-355.

14. Meyer D, Birchmeier C. Multiple essential functions of neuregulin in development. Nature. 1995;378(6555):386-390.
15. Lee KF, Simon H, Chen H, Bates B, Hung MC, Hauser C. Requirement for neuregulin receptor erbB2 in neural and cardiac development. Nature. 1995;378(6555):394-398.

16. Riethmacher D, Sonnenberg-Riethmacher E, Brinkmann V, Yamaai T, Lewin GR, Birchmeier C. Severe neuropathies in mice with targeted mutations in the ErbB3 receptor. Nature. 1997;389(6652):725-730.

17. Chen S, Rio C, Ji RR, et al. Disruption of ErbB receptor signaling in adult non-myelinating Schwann cells causes progressive sensory loss. Nat Neurosci. 2003;6(11):1186-1193.

18. Calvo M, Zhu N, Tsantoulas C, et al. Neuregulin-ErbB signaling promotes microglial proliferation and chemotaxis contributing to microgliosis and pain after peripheral nerve injury. $J$ Neurosci. 2010;30(15):5437-5450.

19. Reinhard S, Vela E, Bombara N, Devries GH, Raabe TD. Developmental regulation of Neuregulin1 isoforms and erbB receptor expression in intact rat dorsal root ganglia. Neurochem Res. 2009;34(1):17-22.

20. Pan B, Huang XF, Deng C. Antipsychotic treatment and neuregulin 1-ErbB4 signalling in schizophrenia. Prog Neuropsychopharmacol Biol Psychiatry. 2011;35(4):924-930.

21. Zhang H, Berezov A, Wang Q, et al. ErbB receptors: from oncogenes to targeted cancer therapies. J Clin Invest. 2007;117(8):2051-2058.

22. Pearson RJ Jr, Carroll SL. ErbB transmembrane tyrosine kinase receptors are expressed by sensory and motor neurons projecting into sciatic nerve. J Histochem Cytochem. 2004;52(10):1299-1311.

23. Matsuoka Y, Yang J. Selective inhibition of extracellular signalregulated kinases $1 / 2$ blocks nerve growth factor to brain-derived neurotrophic factor signaling and suppresses the development of and reverses already established pain behavior in rats. Neuroscience. 2012;206:224-236.
Journal of Pain Research

\section{Publish your work in this journal}

The Journal of Pain Research is an international, peer-reviewed, open access, online journal that welcomes laboratory and clinical findings in the fields of pain research and the prevention and management of pain. Original research, reviews, symposium reports, hypothesis formation and commentaries are all considered for publication.

\section{Dovepress}

The manuscript management system is completely online and includes a very quick and fair peer-review system, which is all easy to use. Visit http://www.dovepress.com/testimonials.php to read real quotes from published authors. 